\title{
Primary central nervous system lymphoma in a rheumatoid arthritis patient treated with methotrexate: a case report
}

Hiromi Shimada ${ }^{1 *}$, Hiroaki Dobashi ${ }^{1}$, Hisanori Morimoto ${ }^{2}$, Tomohiro Kameda $^{1}$, Kentaro Susaki ${ }^{1}$, Miharu Izumikawa ${ }^{1}$, Yohei Takeuchi', Shusaku Nakashima', Osamu Imataki ${ }^{1}$ and Shuji Bandoh'

\begin{abstract}
Background: Rheumatoid arthritis is a systemic inflammatory disease characterized by synovitis and the destruction of articular structures in multiple joints. Methotrexate is recommended as an anchor drug for rheumatoid arthritis treatment to achieve the therapeutic goal of reducing damage to joints and improving clinical score. However, several studies have shown that methotrexate has been associated with the development of lymphoproliferative disorders, namely methotrexate-associated lymphoproliferative disorders. On the other hand, primary central nervous system lymphoma is an aggressive disease with poor prognosis. Both methotrexate-associated lymphoproliferative disorders and primary central nervous system lymphoma are reported to be associated with Epstein-Barr virus.

Case presentation: A Japanese female patient of between 60 and 70 years of age with rheumatoid arthritis was admitted to our hospital because of sudden convulsion and impaired consciousness. Just before admission, she was treated with adalimumab and methotrexate. Contrast-enhanced computed tomography scan showed a densely stained mass with surrounding edema in both frontal lobes and the left nucleus basalis, and enlarged lymph nodes in the right supraclavicular fossa. We performed a biopsy of the right cervical lymph node, but could not establish a histopathological diagnosis. In situ hybridization showed the presence of Epstein Barr virus, therefore we diagnosed this case as methotrexate-associated lymphoproliferative disorders mediated by Epstein Barr virus after considering the drug history of the patient. After we discontinued methotrexate, patient symptoms gradually improved. The masses at both frontal lobes and the left nucleus basalis were gradually regressed.
\end{abstract}

Conclusion: Since the frequency of methotrexate use and the maximum dosage has been increasing, particular attention should be paid to the development of methotrexate-associated lymphoproliferative disorders in rheumatoid arthritis patients who are treated with methotrexate.

Keywords: Rheumatoid arthritis, Methotrexate (MTX), Primary central nervous system lymphoma (PCNSL), MTX-associated lymphoproliferative disorders (MTX-LPD)

\section{Background}

Rheumatoid arthritis (RA) is a systemic inflammatory disease that is characterized by inflammation of the synovial membrane and extensive, progressive destruction of articular structures in multiple joints. The therapeutic goal of RA is to control such tissue damage and to improve long-term prognosis. Methotrexate (MTX) is the

\footnotetext{
* Correspondence: h_kizu@med.kagawa-u.ac.jp

${ }^{1}$ Department of Internal Medicine, Division of Hematology, Rheumatology and Respiratory Medicine, Faculty of Medicine, Kagawa University, 1750-1 Ikenobe, Miki-cho, Kita-gun, Kagawa 761-0793, Japan

Full list of author information is available at the end of the article
}

anchor drug for RA treatment, and is expected to suppress the articular destruction. However, several studies have shown that patients with RA have a high risk of onset of lymphoproliferative disorders (LPD) [1], and MTX has been associated with the development of these disorders [2]. In 2008, according to the World Health Organization (WHO) Classification of Tumours of Haematopoietic and Lymphoid Tissues, LPD induced in immunodeficient patients treated with MTX is specifically defined as MTX-associated LPD (MTX-LPD) [3]. Characteristically, MTX-LPD is reported to regress 
spontaneously following the withdrawal of MTX [4]. Additionally, an association with Epstein-Barr virus (EBV) is indicated [5]. However, primary central nervous system lymphoma (PCNSL) is aggressive and has a poor prognosis [6]. Several reports have indicated that PCNSL is also related to EBV infection, and EBV-related PCNSL occurs more frequently in acquired immunodeficiency syndrome (AIDS) patients and organ transplant recipients with severely decreased immunocompetence [7]. However, EBVrelated PCNSL rarely occurs in patients treated with immunosuppressive therapy. Herein, we describe a RA patient treated with MTX who developed MTX-associated and EBV-related PCNSL.

\section{Case presentation}

A Japanese female patient of between 60 and 70 years of age was admitted to the emergency room in our hospital because of sudden convulsion and impaired consciousness in September 2012. She had been diagnosed with RA two decades earlier. She began MTX therapy in 2005, and etanercept was added the next year, but was then changed to adalimumab (ADA) in 2009. Just before admission to our hospital, she was undergoing treatment with ADA (40 mg/2 weeks), MTX (14 mg/week), and predonisolone (PSL) $(2 \mathrm{mg} /$ day). In the emergency room, her level of consciousness was E1V1M1 on the Glasgow Coma Scale. Additionally, her upper and lower limbs had a convulsive seizure. Although she did not have anisocoria, her eyeballs were deviated to the left side. After we administered diazepam, her convulsions were improved. However, relapse occurred within a short duration, and the patient was therefore admitted to the intensive care unit.

On admission, hematological and biochemical testing (Table 1) revealed an elevated white blood cell count to 14730 per microliter (neutrophils, 64.0\%; lymphocytes, 24.0\%). C-reactive protein and lactate dehydrogenase were also increased to $0.81 \mathrm{mg} / \mathrm{dl}$ (normal range; $<0.2$ $\mathrm{mg} / \mathrm{dl}$ ) and $306 \mathrm{IU} / \mathrm{l}$ (normal range; 110 220 IU/l), respectively. Immunological test results were within almost normal ranges, except for rheumatoid factor (RF; $64 \mathrm{IU} / \mathrm{ml}$, normal range; $<15 \mathrm{U} / \mathrm{ml}$ ). Procalcitonin and beta-D-glucan were negative, and ferritin and soluble interleukin-2 receptor were within normal ranges. EBV antibody showed a pattern indicative of previous infection (EB-VCA-IgG, 160 titer; EBV-VCA-IgM, negative; anti-EBNA, 80 titer). Cranial computed tomography (CT) scan showed low density areas in the frontal lobes of both sides and the nucleus basalis of the left side (Figure 1). Convulsions disappeared following repeated administration of diazepam.

Table 1 Laboratory data upon hospital admission

\begin{tabular}{|c|c|c|c|c|c|c|c|c|c|c|c|}
\hline \multicolumn{3}{|l|}{ CBC } & \multicolumn{3}{|c|}{ Biochemistry } & \multicolumn{3}{|c|}{ Immunology } & \multicolumn{3}{|l|}{ Infection } \\
\hline WBC & 14730 & $/ \mu l$ & CRP & 0.81 & $\mathrm{mg} / \mathrm{dl}$ & $\lg G$ & 1335 & $\mathrm{mg} / \mathrm{dl}$ & PCT & $<0.02$ & $\mathrm{ng} / \mathrm{ml}$ \\
\hline \multirow[t]{2}{*}{ Neu. } & 64 & $\%$ & $\mathrm{TP}$ & 7.7 & $\mathrm{~g} / \mathrm{dl}$ & $\lg A$ & 113 & mg/dl & $\beta-D$ & $<5.0$ & $\mathrm{pg} / \mathrm{ml}$ \\
\hline & & & & & & & & & glucan & & \\
\hline \multirow[t]{2}{*}{ Lym. } & 24 & $\%$ & Alb & 4.4 & $\mathrm{~g} / \mathrm{dl}$ & $\lg M$ & 103 & mg/dl & EBV-VCA & 0.15 & \\
\hline & & & & & & & & & $\lg G$ & & \\
\hline \multirow[t]{2}{*}{ Mono. } & 11.5 & $\%$ & BUN & 13 & mg/dl & $\mathrm{C} 3$ & 155.9 & mg/dl & EBV-VCA & negative & \\
\hline & & & & & & & & & $\lg M$ & & \\
\hline Eos. & 0.5 & $\%$ & $\mathrm{Cr}$ & 0.6 & mg/dl & C4 & 27.2 & mg/dl & EBNA & 0.1 & \\
\hline Baso. & 0 & $\%$ & eGFR & 75.99 & ml/min. & $\mathrm{CH} 50$ & 63.8 & $\mathrm{U} / \mathrm{ml}$ & T-SPOT Tb & negative & \\
\hline $\mathrm{RBC}$ & 391 & $\times 104 / \mu l$ & T-bil & 0.7 & mg/dl & ANA & 1:40, Ho. & & & & \\
\hline $\mathrm{Hb}$ & 12.3 & $\mathrm{~g} / \mathrm{dl}$ & AST & 25 & IU/I & RF & 64 & IU/ml & & & \\
\hline $\mathrm{Hct}$ & 36.8 & $\%$ & ALT & 12 & IU/I & MMP-3 & 33.2 & $\mathrm{ng} / \mathrm{ml}$ & $\mathrm{ABG}(\mathrm{O} 2 ; 3 \mathrm{~L})$ & & \\
\hline \multirow[t]{9}{*}{ Plt } & 25.8 & $\times 104 / \mu l$ & ALP & 246 & IU/I & SS-A Ab & 10.1 & $\mathrm{U} / \mathrm{ml}$ & $\mathrm{pH}$ & 7.29 & \\
\hline & & & YGTP & 27 & IU/I & SS-B Ab & 8.9 & $\mathrm{U} / \mathrm{ml}$ & $\mathrm{pCO} 2$ & 49 & Torr \\
\hline & & & $\mathrm{LDH}$ & 306 & IU/I & MPO- & $<3.0$ & IU/ml & pO2 & 74 & Torr \\
\hline & & & & & & ANCA & & & & & \\
\hline & & & $\mathrm{Na}$ & 143 & $\mathrm{mEq} / \mathrm{l}$ & PR3- & $<3.5$ & IU/ml & $\mathrm{HCO} 3$ & 24.3 & $\mathrm{mmol} / \mathrm{l}$ \\
\hline & & & & & & ANCA & & & & & \\
\hline & & & K & 4.4 & $\mathrm{mEq} / \mathrm{l}$ & sIL-2R & 391 & $\mathrm{U} / \mathrm{ml}$ & $\mathrm{BE}$ & -3.4 & $\mathrm{mmol} / \mathrm{l}$ \\
\hline & & & $\mathrm{Cl}$ & 105 & $\mathrm{mEq} / \mathrm{l}$ & & & & $\mathrm{SpO} 2$ & 94.8 & $\%$ \\
\hline & & & $\mathrm{Ca}$ & 9.3 & $\mathrm{mEq} / \mathrm{l}$ & & & & & & \\
\hline
\end{tabular}





Figure 1 Cranial computed tomography scan on admission. Arrows indicated low density areas at the frontal lobes of both sides and the nucleus basalis of left side.

However, we performed artificial ventilation because of severe respiratory depression. Additionally, we discontinued MTX and ADA, and increased PSL dose to $10 \mathrm{mg} /$ day.

On the sixth day after admission, we performed a contrast-enhanced CT scan, which showed a densely stained mass with surrounding edema in the left frontal lobes and nucleus basalis, and enlarged lymph nodes in the right supraclavicular fossa (Figure 2). Furthermore, the accumulation in swollen lymph nodes was detected on fluorodeoxyglucose (FDG) positron emission tomography (Figure 3). Therefore, we performed a biopsy of the right cervical lymph node.

Histopathological examination of the right cervical lymph node showed the disappearance of follicle structure and dispersed Hodgkin-like large cells with small lymphocytes. These large cells were positive for CD30, Bob1 and Oct2, undeterminable for CD20 and PAX5, and negative for CD15. This finding differed from typical Hodgkin lymphoma, which generally has immunoreactivity for CD30, CD15, and PAX5 (Figure 4 a-e). Many of the small lymphocytes were CD20-positive cells. However, these were negative for MIB-1, and IgH reconstitution was not observed by polymerase chain reaction, therefore we could not diagnose this case as B-cell lymphoma. In situ hybridization showed EBV-encoded ribonucleic acid (EBER) in the nuclei of lymphoma cells (Figure 4f). Consequently, we could not establish a histopathological diagnosis and therefore diagnosed this case as MTX-associated LPD after considering the drug history of the patient.

After withdrawal of MTX, patient symptoms gradually improved. The masses at both frontal lobes and the left nucleus basalis, and the lymphadenopathy in the right supraclavicular fossa were gradually reduced, as observed by CT scan (Figure 5) and there was no accumulation of
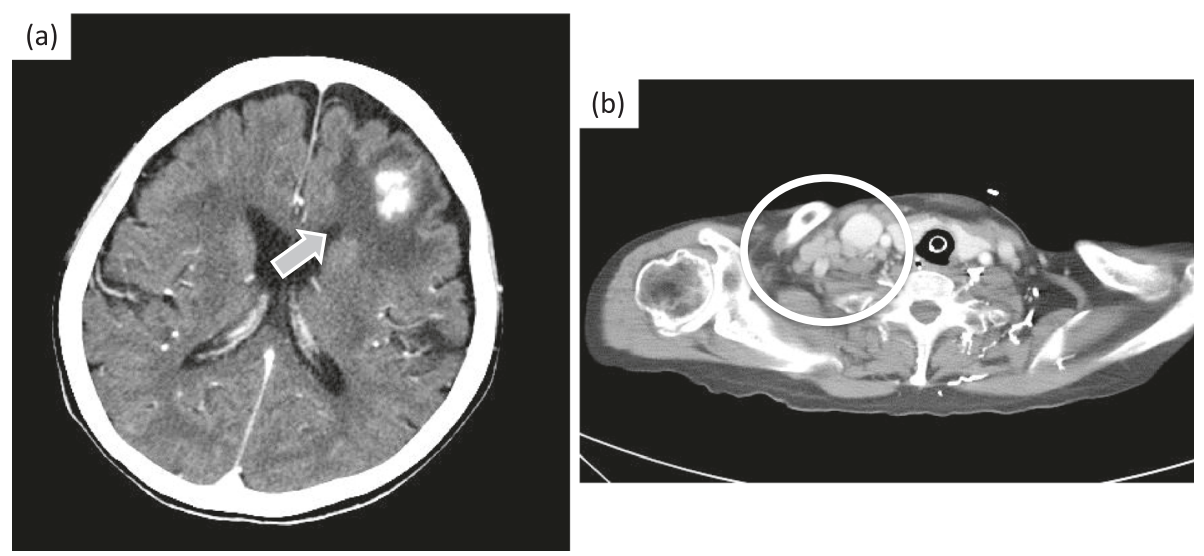

Figure 2 Contrast-enhanced computed tomography scan on the sixth day after admission. (a) Arrow indicated showed densely stained masses with surrounding edema at the left frontal lobe, and (b) circle showed many swollen lymph nodes in the right supraclavicular fossa. 


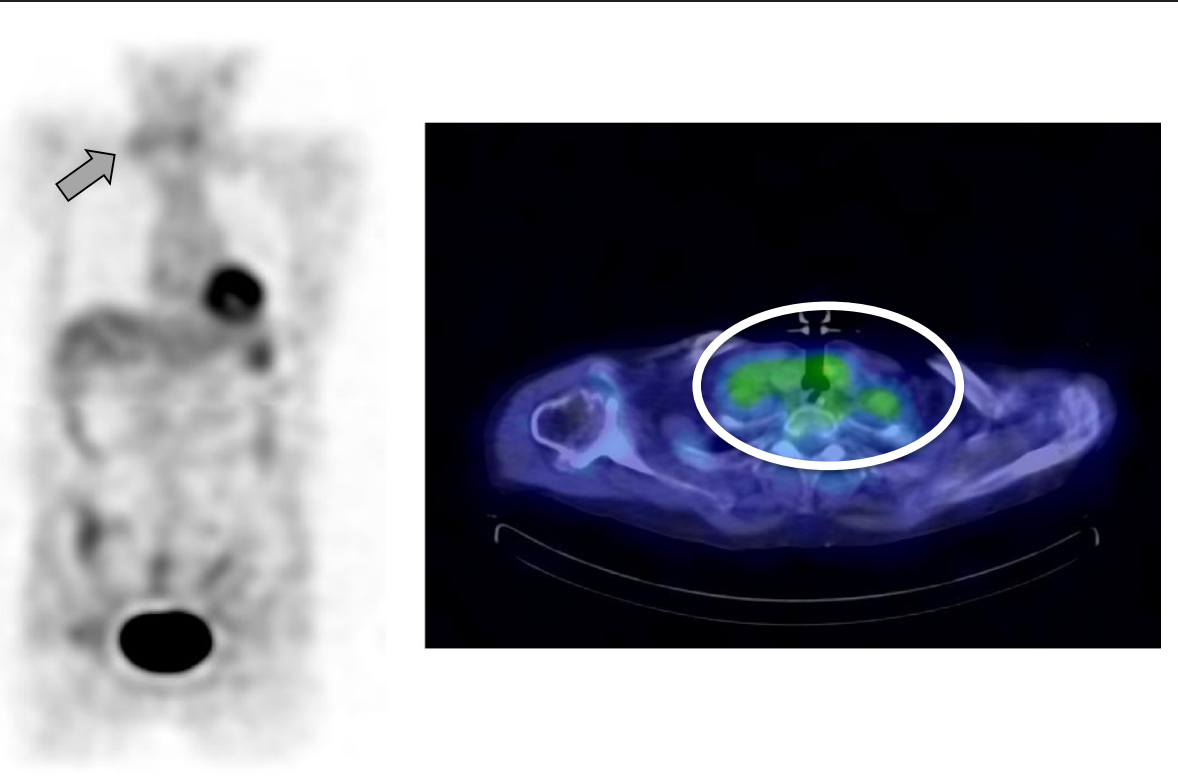

Figure 3 Fluorodeoxyglucose positron emission tomography/computed tomography scan. Arrow and circle indicated the accumulation of fluorodeoxyglucose in the swollen lymph nodes of the right supraclavicular fossa.
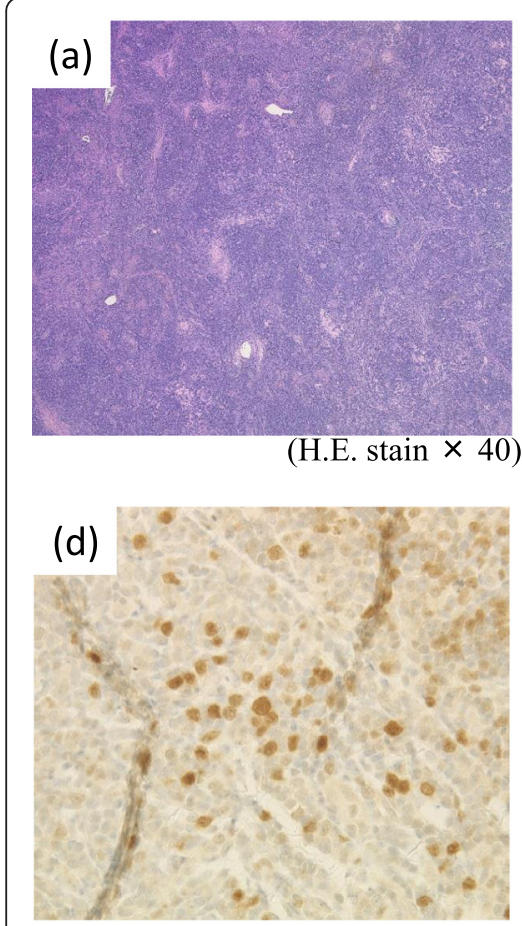

$($ Bob1 $\times$ 400)
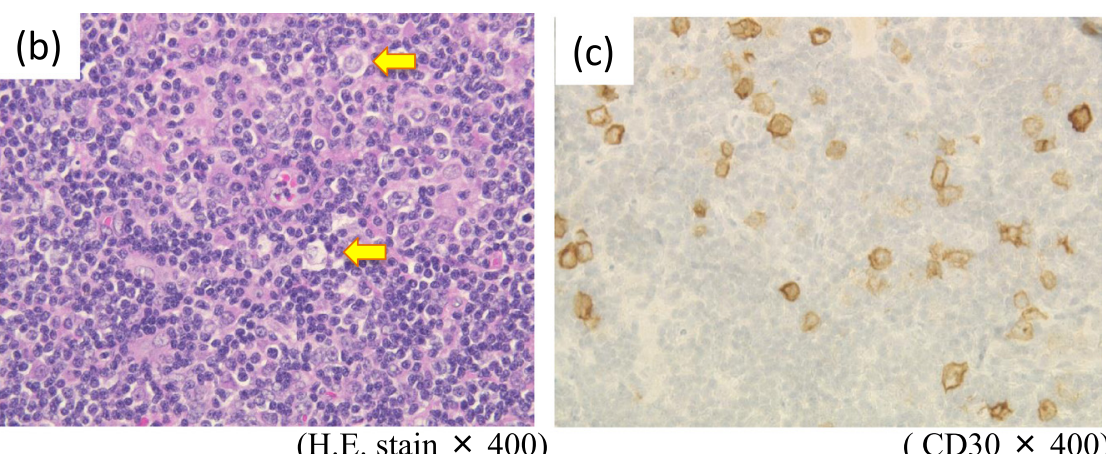

(H.E. stain $\times 400)$



(f)

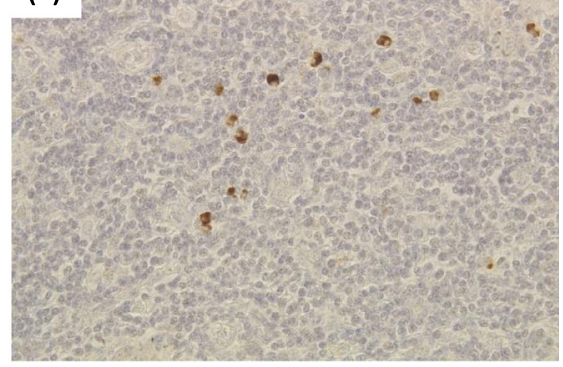

$($ EBER $\times 400)$

Figure 4 Biopsy of swollen lymph nodes in the right supraclavicular fossa. Hematoxylin and eosin staining showed (a) the disappearance of follicle structure, and (b) dispersed Hodgkin-like large cells with small lymphocytes. (c)-(e) Immunostaining for CD30, Bob1 and Oct2 showed positive large cells. (f) In situ hybridization showed Epstein-Barr virus encoded ribonucleic acid in the nuclei of lymphoma cells. 


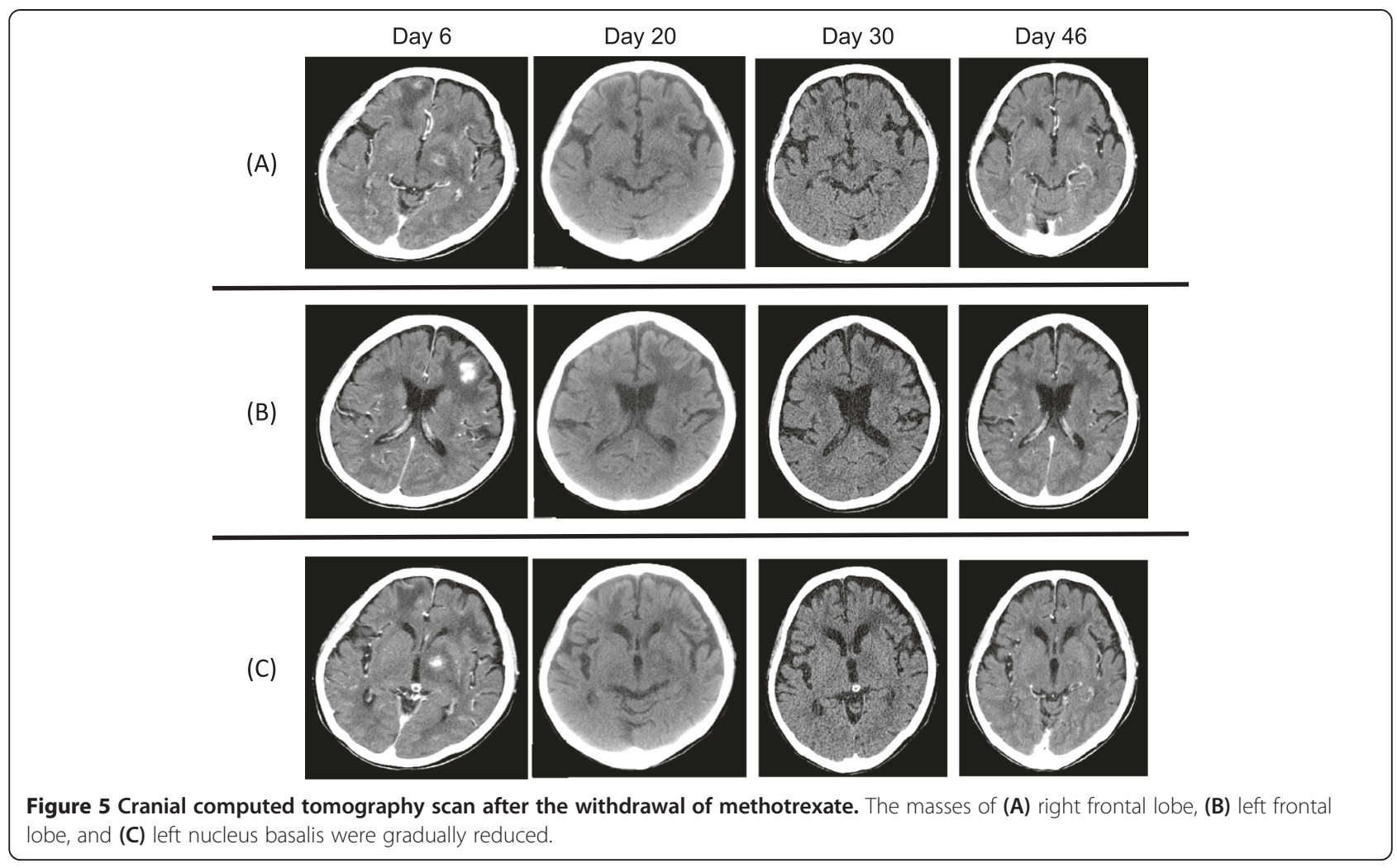

FDG after 3 months. The patient was treated with PSL and salazosulfapyridine (1000 $\mathrm{mg} /$ day) for RA, and no recurrence of lymphoma was observed more than one year after the discontinuation of MTX.

\section{Discussion}

MTX is an important drug used for the treatment of RA. Hence, over time, the frequency of MTX use and the maximum dosage has been increasing. However, several studies have reported that LPD more readily develops in immunodeficient patients treated with MTX [2]. The characteristics of MTX-LPD are that 1) lymphoma regresses spontaneously following the withdrawal of MTX treatment [4], 2) MTX-LPD is associated with EBV seropositivity [5], and 3) histological findings show various tissue types including diffuse large B-cell lymphoma, Hodgkin lymphoma, polymorphic-LPD, and Hodgkin like-lymphoma [8].

EBV is an oncogenic virus associated with several malignant diseases such as Burkitt's lymphoma, nonHodgkin lymphoma, and so on [9]. These malignancies are usually considered to be the result of virus reactivation [10]. EBV was demonstrated in approximately $40 \%$ of MTX-LPD cases, and most of them regress spontaneously [3]. The mechanism of MTX-LPD is that the immunosuppressive state induced by MTX treatment may allow reactivation of EBV as normal anti-virus surveillance is impaired, and some MTX-LPDs associated with
EBV may regress after MTX withdrawal by the restoration of normal immunity [4].

In our case, a RA patient with a history of MTX administration had a sudden convulsion, multiple brain tumors, and lymphadenopathy observable by CT scan. We could not make a definite diagnosis by histological findings of cervical lymph node biopsy, which demonstrated Hodgkin-like large cells and many CD20-positive lymphocytes. Additionally, EBERs were detected in the nuclei of lymphoma cells. Therefore, we diagnosed MTXLPD mediated by EBV.

However, we could perform the biopsy not from central nervous system (CNS) lesion but from cervical lymph node. In fact, we could not judge whether the CNS tumor was MTX-LPD or not. After withdrawal of MTX, symptoms gradually improved, and both the CNS tumors and cervical lymphadenopathy remarkably decreased, as observed by cranial CT scan. Hence, we could conclude that these tumors are from same origin.

In this case, ADA was also administered but discontinued after onset of convulsions, concomitantly with MTX. However, Ramiro et al. indicated that patients treated with an anti-tumor necrosis factor (TNF) inhibitor did not have an increased risk of lymphoma [11]. Burmester et al. showed that the greater rate of lymphoma occurrence observed during ADA trials in patients with RA was within the range that might be expected in a similar RA population not treated with anti-TNF therapy [12]. Therefore, in the 
present case study, we considered the lymphoma to be caused by MTX and not ADA.

PCNSL occurs in brain, spinal cord, eyeball, cranial nerve, and meninges without affecting other organs. It is aggressive, and almost always of B-cell origin [6]. Although PCNSL is a rare lymphoma that accounts for only approximately $3 \%$ of all primary brain tumors, the incidence tends to increase within the last 10 years. High-dose MTX and radiation therapy is recommended as a treatment of PCNSL [13]. Furthermore, previous reports uphold the relationship between EBV and PCNSL [10,14-16]. In general, EBV-related PCNSL has a poor prognosis. Moreover, it occurs in some AIDS patients, organ transplant recipients and a few immunocompetent individuals [7]. Furthermore, there are a few reports that PCNSLs occurred in patients treated with immunosuppressive drugs. Kleinschmidt-DeMasters et al. showed four cases of EBV-related PCNSL occurring in older patients who were treated with immunosuppressive drugs (MTX, mycophenolate mofetil, azathioprine, PSL, cyclophosphamide, and so on). In one case, in which the patient was treated with MTX, the lymphoma improved following the cessation of MTX and specific treatment for the toxoplasmosis [17]. Her neurological deficits markedly improved, but she developed renal failure and died on approximately 3 months later. Migita et al. also reported a patient with RA who developed an EBV-related PCNSL during treatment with MTX. They underwent surgical resection and MTX withdrawal, resulting in MTXLPD improvement without any recurrence [18].

In our case, PCNSL markedly regressed only by the discontinuation of MTX, and there is presently no recurrence. This finding confirms the potentiality of the spontaneous nature of MTX-LPD regression following cessation of MTX treatment.

\section{Conclusion}

We have presented a rare case in which a RA patient treated with MTX developed MTX-associated, EBVrelated PCNSL, which regressed markedly upon the withdrawal of MTX. Since current treatment strategies for RA recommend MTX as a first choice therapy in early stages of disease, the frequency of MTX use and the maximum dosage has been increasing. Therefore, we need to pay attention to the development of MTXLPD for RA patients who are treated with MTX.

\section{Consent}

Written informed consent was obtained from the patient for publications of this Case Report and any accompanying images. A copy of the written consent is available for review by the Editor-in-Chief of the journal.

\section{Abbreviations}

RA: Rheumatoid arthritis; MTX: Methotrexate; LPD: Lymphoproliferative disorder; WHO: World Health Organization; MTX-LPD: Methotrexate-related lymphoproliferative disorder; PCNSL: Primary central nerve system

lymphoma; AIDS: Acquired immunodeficiency syndrome; EBV: Epstein-Barr virus; ADA: Adalimumab; PSL: Predonisolone; RF: Rheumatoid factor; $C T$ : Computed tomography; FDG: Fluorodeoxyglucose; EBER: Epstein-Barr virus encoded ribonucleic acid; CNS: Central nervous system; TNF: Tumor necrosis factor.

\section{Competing interests}

The authors declare that they have no competing interests.

\section{Authors' contributions}

$H S, H D, T K, K S, M I, S N$, and YT made substantial contributions to conception and acquisition of data and analysis and interpretation of data. HM and Ol helped to draft the manuscript. All authors read and approved the final manuscript.

\section{Acknowledgements}

The authors do not have any acknowledgement to make. None of the authors were recipients of any sources of funding and no funding was received towards the preparation of the manuscript.

\section{Author details}

'Department of Internal Medicine, Division of Hematology, Rheumatology and Respiratory Medicine, Faculty of Medicine, Kagawa University, 1750-1 Ikenobe, Miki-cho, Kita-gun, Kagawa 761-0793, Japan. ${ }^{2}$ Department of Internal Medicine, Mitoyo general hospital, 708 Himehama, Toyohama-cho, Kanonji-si, Kagawa 769-1695, Japan.

Received: 25 September 2014 Accepted: 3 March 2015

Published online: 19 March 2015

\section{References}

1. Zintzaras E, Voulgarelis M, Moutsopoulos HM. The risk of lymphoma development in autoimmune diseases: a meta-analysis. Arch Intern Med. 2005;165:2337-44.

2. Mariette X, Cazals-Hatem D, Warszawki J, Liote F, Balandraud N, Sibilia J, et al. Lymphomas in rheumatoid arthritis patients treated with methotrexate: a 3-year prospective study in France. Blood. 2002;99:3909-15.

3. Swerdlow SH, Campo E, Harris NL, Jaffe ES, Pileri SA, Stein H, et al. WHO Classification of Tumors of the Haematopoietic and Lymphoid Tissues. Lyon: IARC Press; 2008.

4. Salloum E, Cooper DL, Howe G, Lacy J, Tallini G, Crouch J, et al. Spontaneous regression of lymphoproliferative disorders in patients treated with methotrexate for rheumatoid arthritis and other rheumatic diseases. J Clin Oncol. 1996;14:1943-9.

5. Feng WH, Cohen Jl, Fischer S, Li L, Sneller M, Goldbach-Mansky R, et al. Reactivation of latent Epstein-Barr virus by Methotrexate: a potential contributor to methotrexate-associated lymphomas. J Natl Cancer Inst. 2004;96:1691-702.

6. Fine HA, Mayer RJ. Primary central nervous system lymphoma. Ann Intern Med. 1993;119:1093-104.

7. Batara JF, Grossman SA. Primary central nervous system lymphomas. Curr Opin Neurol. 2003;16:671-5.

8. Hoshida Y, Xu J-X, Fujita S, Nakamichi I, Ikeda JI, Tomita Y, et al. Lymphoproliferative disorders in rheumatoid arthritis: clinicopathological analysis of 76 cases in relation to methotrexate medication. J Rheumatol. 2007;34:322-31.

9. Hsu JL, Glaser SL. Epstein-Barr virus-associated malignancies: epidemiologic patterns and etiologic implications. Crit Rev Oncol Hematol. 2000;34:27-53.

10. Gulley ML. Molecular diagnosis of Epstein-Barr virus-related diseases. J Mol Diagn. 2001;3:1-10.

11. Ramiro S, Gaujoux-Viala C, Nam JL, Smolen JS, Buch M, Gossec L, et al. Safety of synthetic and biological DMARDs: a systematic literature review informing the 2013 update of the EULAR recommendations for management of rheumatoid arthritis. Ann Rheum Dis. 2014;73:529-35.

12. Burmester GR, Panaccione R, Gordon KB, Mcllraith MJ, Lacera AP. Adalimumab: long-term safety in 23458 patients from global clinical trials in rheumatoid arthritis, juvenile idiopathic arthritis, ankylosing spondylitis, 
psoriatic arthritis, psoriasis and Crohn's disease. Ann Rheum Dis. 2013;72:517-24.

13. Korfel A, Schlegel U. Diagnosis and treatment of primary CNS lymphoma. Nat Rev Neurol. 2013;9:317-27.

14. Geddes JF, Bhattacharjee MB, Savage K, Scaravilli F, McLaughlin JE. Primary cerebral lymphoma: a study of 47 cases probed for Epstein-Barr virus genome. J Clin Pathol. 1992;45:587-90.

15. Hamilton-Dutoit SJ, Raphael M, Audouin J, Diebold J, Lisse I, Pedersen C, et al. In situ demonstration of Epstein-Barr virus small RNAs (EBER-1) in acquired immunodeficiency syndrome-related lymphomas: correlation with tumor morphology and primary site. Blood. 1993;82:619-24.

16. Krogh-Jensen $M$, Johansen $P, D^{\prime}$ Amore F. Primary central nervous system lymphomas in immunocompetent individuals: histology, Epstein-Barr virus genome, Ki-67 proliferation index, p53 and bcl-2 gene expression. Leuk Lymphoma. 1998;30:131-42.

17. Kleinschmidt-DeMasters BK, Damek DM, Lillehei KO, Dogan A, Giannnini C. Epstein-Barr virus-associated primary CNS lymphomas in elderly patients on immunosuppressive medications. J Neouropathol Exp Neurol. 2008:67:1103-11.

18. Migita K, Miyashita T, Mijin T, Sakito S, Kurohama H, Ito M, et al. Epstein-Barr virus and methotrexate-related CNS lymphoma in a patient with rheumatoid arthritis. Mod Rheumatol. 2013;23:832-6.

\section{Submit your next manuscript to BioMed Central and take full advantage of:}

- Convenient online submission

- Thorough peer review

- No space constraints or color figure charges

- Immediate publication on acceptance

- Inclusion in PubMed, CAS, Scopus and Google Scholar

- Research which is freely available for redistribution 